\title{
In Memoriam Herbert J. Nickel
}

(Würzburg, Alemania 1935-Bayreuth, Alemania 2020)

₹

In Memoriam Herbert J. Nickel

(Würzburg, Germany 1935-Bayreuth, Germany 2020)

María Eugenia Ponce Alcocer

Universidad Iberoamericana

México

Correo: mponcealcocer@gmail.com

https://orcid.org/0000-0002-9437-388I

DOI: 10.48102/hyg.vi56.376

Artículo recibido: 4/09/2020

Artículo aceptado:9/10/2020

Mi objetivo era basar las investigaciones en fuentes empíricas, en el caso de estudios sobre la hacienda mexicana: en libros de contabilidad, la correspondencia de dueños y administradores, documentos sobre títulos de tierra, documentos sobre conflictos jurídicos y protocolos de juzgados. [...] la investigación empírica es un ejercicio fascinante, si bien no siempre produce resultados convincentes, por la falta de datos empíricos suficientes. ${ }^{1}$

Esta cita nos presenta en pocas líneas la síntesis de la metodo-
logía de Nickel para investigar la institución que conocemos como hacienda, y se reconoce, además, el problema que se presenta ante cualquier investigación, por la insuficiencia de datos empíricos.

${ }^{1}$ Herbert J. Nickel, Apuntes realizados para un video para la presentación del doctor Nickel en la ceremonia de premiación Reconocimiento al Investigador Extranjero del XVIII Premio Citibanamex Atanasio G. Saravia de Historia Regional Mexicana 2018-2019.

Historia y Grafia, Universidad Iberoamericana, año 28, núm. 56, enero-junio 2021, pp. 389-397 
Herbert J. Nickel realizó estudios como ingeniero topógrafo en la Staatsbauschule München, y después comenzó a estudiar Economía, Geografía y Sociología en la Universidad de Erlangen-Nuremberg, pero interrumpió la carrera, para trabajar como topógrafo en el proyecto de la cuenca Puebla-Tlaxcala de la Fundación Alemana para la Investigación Científica (Deutsche Akademische Austausch Dienst DAAD,1966). Allí se encargó de hacer las mediciones y levantamientos topográficos de los restos prehispánicos, y la Fundación le concedió tiempo para estudiar dos temas de su preferencia: a los pueblos de indígenas que habían sido trasladados más al centro del territorio con el propósito de lograr un mejor control por parte de la administración novohispana, y una monografía sobre la hacienda Amalucan, cercana a la ciudad de Puebla. Allí nació su interés por el estudio de esta unidad económica y social muy importante en la historia agraria de México, pero sobre todo comenzaron sus dudas sobre el papel de esta institución en la historia oficial mexicana.

En 1967 fue admitido como graduado en la Universidad de Berkeley en el instituto de Geografía. Su graduate advisor lo mandó a que visitara al Prof. Woodrow Borah, quien le recomendó escribir una tesis sobre una hacienda. Al mismo tiempo recibió la invitación del profesor doctor Dieter Oberndörfer, para continuar sus estudios en la Universidad Friburgo, y optó por regresar a Alemania. Posteriormente obtuvo su Doctorado en Sociología con segunda especialidad en geografía y etnología en la mencionada Universidad, donde también hizo su doctorado por habilitación con la tesis "Morfología social de la hacienda mexicana" (1977), que fue publicada en alemán un año después. Se trata de un excelente estudio que "conforma interpretación teórica, el estudio regional y el monográfico", decía Gisela von Wobeser al hacer la primera reseña en español sobre el libro de Nickel. ${ }^{2}$

${ }^{2}$ Gisela von Wobeser, "Reseña de Morfología social de la hacienda mexicana", Boletín de Información del Instituto de Investigaciones Históricas, UNAM, mayo-agosto de 1980 , p. 15. 
Para Nickel, las haciendas fueron grandes explotaciones agrícolas que tuvieron su política y sus tácticas de mercado y funcionaron como empresas capitalistas. Su obra derriba el "viejo e inoperante cliché de la hacienda clásica que Molina Enríquez y la historiografía revolucionaria grabaron y agravaron enalteciendo la reforma agraria revolucionaria”. 3

En 1983 estuvo por primera vez como profesor en el Departamento de Historia de la Universidad Iberoamericana para impartir un seminario sobre Relaciones de trabajo en las haciendas. Fue invitado con el propósito de fortalecer la línea de investigación orientada a la historia agraria, iniciada por la maestra Beatriz Ruiz Gaytán, quien antes había llamado al doctor Francisco de Solano y Pérez Lila (impartió un curso sobre historia de la propiedad agraria), y posteriormente al doctor Moisés González Navarro (dio un curso sobre haciendas). Además, la Universidad había adquirido un archivo sobre dos haciendas en Tlaxcala: Mazaquiahuac y El Rosario y otra en el Estado de México: San Nicolás del Moral, con el fin de que fuera utilizado por los alumnos del Departamento.

En 1987 fue publicada por la Iberoamericana su obra Relaciones de trabajo en las haciendas de Puebla y Tlaxcala, 1740-1914: cuatro análisis sobre reclutamiento, peonaje y remuneración. Continuó sus investigaciones con libros de contabilidad, de correspondencia de haciendas, archivos notariales, en archivos públicos como el Archivo General de la Nación, el Archivo del Estado de Yucatán y el de Chihuahua, archivos de haciendas en la Universidad Autónoma de Puebla y privados como los de la Biblioteca de la Universidad Iberoamericana, más archivos particulares de diversas haciendas en los estados de Puebla, Tlaxcala, Chihuahua y Yucatán.

\footnotetext{
${ }^{3}$ Ursula Ewald, "Book Reviews", The Americas, A Quartely Review of Inter American Cultural History, Academy of American Franciscan History, vol. 36, núm. 4 (April 1980), p. 552; Heriberto Moreno, "Nickel: La hacienda ¿dominio o coexistencia?”, Relaciones, núm. 39, p. 121 (115-137).
} 
Resultado de su segundo seminario en la Universidad Iberoamericana fue el libro Paternalismo y economía moral en las haciendas mexicanas del Porfiriato, ${ }^{4}$ en el que, junto con sus alumnos Ricardo Rendón, Marisa Pérez Domínguez y María Eugenia Ponce, así como Hans Günter Mertens, expusieron cómo la economía moral se puso en práctica en las haciendas mexicanas en las relaciones de trabajo, a través de las prestaciones como la asistencia médica, adelantos en efectivo y en especie, y pensiones a las viudas, que el hacendado otorgó a sus trabajadores. Con esta publicación muchos lectores mexicanos conocieron los principales conceptos propuestos por el modelo de la "economía moral" desarrollado -entre otros- por James Scott, Eric Wolf, Samuel Popkin y Edward P. Thompson. ${ }^{5}$

Estos seminarios formaron a un grupo de historiadores que se dedicaron a estudiar a la hacienda, institución muy importante en la historia agraria de nuestro país por casi cuatro siglos (del xvI a la cuarta década del xx), como Ricardo Rendón, autor de varias obras que se enfocaron en algunos elementos de la hacienda: cómo funcionó esta institución en producción, comercialización y las relaciones laborales, cómo se vivía en la hacienda en el día a día, también cómo fue percibida la Revolución desde la administración de dos haciendas. ${ }^{6}$ También Marisa Pérez de Sarmiento

${ }^{4}$ Paternalismo y economia moral en las haciendas mexicanas del porfiriato, Herbert J. Nickel (ed.), México, Universidad Iberoamericana, Departamento de Historia, 1989.

${ }^{5}$ James Scott, The Moral Economy of the Peasant Rebellion and Subsistence in Southeast Asia, New Haven/Londres, Yale University Press, 1976; Eric Wolf, Los campesinos, Barcelona, Editorial Labor, 1975; Samuel Popkin, The Rational Peasant. The Political Economy of the Rural Society in Vietnam, Los Angeles, University of California Press, 1979; Edward P. Thompson, "La economía moral de la multitud en la Inglaterra del siglo xvin", Tradición, revuelta y conciencia de clase. Estudios sobre la crisis de la sociedad preindustrial, Barcelona, Editorial Crítica, 1984.

${ }^{6}$ Ricardo Rendón, Dos haciendas pulqueras en Tlaxcala 1867-1884, Tlaxcala, Talleres Gráficas del Estado de Tlaxcala, 1990; Id., Haciendas de México, México, Fomento Cultural Banamex, 1994; Id., La vida cotidiana en las haciendas, 
estudió los servicios médicos que se dieron en algunas haciendas mexicanas. ${ }^{7}$ María Eugenia Ponce, por su parte, trabajó las relaciones laborales, su jerarquía, los trabajadores dedicados a la administración de la finca, sus salarios, prestaciones, endeudamiento, así como la modernización de algunas haciendas durante el régimen de Porfirio Díaz. ${ }^{8}$

México, Fondo Cultural Banamex, 1997; Id., "La revolución armada vista por el administrador de dos haciendas tlaxcaltecas (1910-1920)", Anuario de Humanidades, Universidad Iberoamericana Ciudad de México, núm. viI, 19811983, pp. 183-211; Mariana Yampolsky y Ricardo Rendón, Haciendas poblanas, México, Universidad Iberoamericana-Departamento de Historia, 1992; Ricardo Rendón, "Aportación al estudio de las relaciones económico-morales entre hacendados y trabajadores. El caso de dos haciendas pulqueras en Tlaxcala", en Herbert J. Nickel (ed.), Paternalismo y economia moral en las haciendas mexicanas del porfiriato, México, Universidad Iberoamericana-Departamento de Historia, 1989, pp. 69-92; Id., "Las relaciones laborales en las haciendas pulqueras desde la perspectiva del modelo de la economía moral”, en María Teresa Jarquín Ortega et al. (coords.), Origen y evolución de la hacienda en México: siglos XVI al XX, Memorias del Simposio realizado del 27 al 30 de septiembre de 1989, Toluca, El Colegio Mexiquense/Universidad Iberoamericana/Instituto Nacional de Antropología e Historia, 1990, pp. 151-157.

${ }^{7}$ Marisa Pérez, "Los servicios médicos en las haciendas a finales del siglo XIX: el caso de San Bartolomé de los Tepetates", México, tesis para obtener la licenciatura en Historia, Universidad Iberoamericana, 1986; Id., "Los servicios médicos en las haciendas: un ejemplo en San Bartolomé de los Tepetates", en Herbert J. Nickel (ed.), Paternalismo y economía moral en las haciendas mexicanas del porfiriato, México, Universidad Iberoamericana-Departamento de Historia, 1989, pp. 93-120; Id., "Mecanismos y traslado de trabajadores a Yucatán en el auge henequenero", en María del Rosario Rodríguez Díaz (coord.), El Caribe entre México y Estados Unidos, Morelia, Mich., Universidad Michoacana de San Nicolás de Hidalgo-Instituto de Investigaciones Históricas, 2005, pp. 23-64.

${ }^{8}$ Ricardo Rendón y María Eugenia Ponce, "La hacienda de San Nicolás del Moral. Proceso de formación territorial y análisis económico del ańo 1883”, en La ciudad y el campo en la historia de México, Memoria de la VII Reunión de Historiadores mexicanos y norteamericanos (Oaxaca, 23-26 octubre de 1985), México, Universidad Nacional Autónoma de México, 1992, vol. 2, pp. 573-599; María Eugenia Ponce, "Las relaciones de trabajo de los meseros de la administración en las haciendas porfiristas", Historia y Grafía, núm. 5, 1995, pp. 81-118; Id., "La modernización en algunas haciendas mexicanas: el fin del sistema tradicional: 1867-1920”, Historia y Grafía, núm. 13, 1999, pp. 93-112; Id., "¿Zapatismo en Tlaxcala? El caso de las haciendas de Mazaquiahuac y El Rosario”, Zapatismo: Origen e historia, México, Secretaría de Gobernación/Ins- 
Nickel continuó investigando sobre las relaciones laborales en las haciendas y en especial sobre el peonaje en su "patria chica": Yucatán, lugar al que continuó viniendo hasta que su salud se lo impidió, tanto para continuar sus trabajos de investigación en archivos públicos y privados, como para fotografiar la maquinaria empleada en las haciendas henequeneras. Resultado de esas investigaciones fueron las publicaciones: "Las deudas de los sirvientes en las haciendas henequeneras de Yucatán"; El peonaje en las haciendas mexicanas: interpretaciones, fuentes, hallazgos, y Henequén-Plantagen in Yucatán, Vom ende einer agroindustriellen Monokultur in Mexiko Eine Dokumentation in Bildern (Henequen plantations in Yucatan: the end of an agro-industrial monoculture in Mexico an illustrated documentation). 9

El doctor Nikel combinó estos trabajos con sus investigacio-

tituto Nacional de Estudios Históricos de las Revoluciones de México, 2009, pp. 439-458; Id., "Dos huelgas en dos haciendas mexicanas, 1893 y 1911", en María Eugenia Ponce e Isabel Martínez Ateca (coords.), Testigos del pasado. 30 años de Acervos Históricos, México, Universidad Iberoamericana, 2014, pp. 179-195; Id., "Fuentes para el patrimonio agroindustrial: el uso de la maquinaria en algunas haciendas mexicanas", en Belem Oviedo, Gracias Dorel-Ferré y Mariano Torres Bautista (coords.), Patrimonio agroindustrial. Trayectorias, retos y significados, México, Archivo Histórico y Museo de Minería, A. C. у тіссн Comité Internacional para la Conservación del Patrimonio Industrial México, 2018, pp. 219-236.

${ }^{9}$ Herbert J. Nickel y María Eugenia Ponce, Hacendados y trabajadores agrícolas ante las autoridades: conflictos laborales a fines de la época colonial documentados en el Archivo General de Indias, México, Universidad Iberoamericana-Departamento de Historia, 1996; Herbert J. Nickel, "Las deudas de los sirvientes en las haciendas henequeneras de Yucatán”, Jahrbuch fur Geschichte von Staat, Wirtschaft und Gesellschaft Lateinamerikas, 33, 1996, pp. 313-361; Id., El peonaje en las haciendas mexicanas: interpretaciones, fuentes, hallazgos, México/ Freiburg, Universidad Iberoamericana/Arnold Bergstraesser Institut, 1997; Id., Henequén-Plantagen in Yucatán, Vom ende einer agroindustriellen Monokultur in Mexiko Eine Dokumentation in Bildern [original], Freiburg i. Br., Germany, Arnold Bergstraesser Institute, 2006, fotos (Henequen Plantations in Yucatan: The End of an Agro-industrial Monoculture in Mexico an Illustrated Documentation), translation by Suzanne Le Mière and Jessica Morris; traducción al español Sefi Alonso Bada y Jairo Montobbio Görlich. Freiburg, Germany, Arnold Bergstraesser Institute, 2006. 
nes sobre la medición de haciendas con base en las Ordenanzas de tierras de José Sanz de Escobar y publicó "Joseph Sáenz de Escobar y su tratado sobre geometría práctica y mecánica: Un manual sobre geometría aplicada para personas no cualificadas en la materia escrito en Nueva Espańa (México) alrededor del ańo 1700 "10 con la cartografía histórica "Kartographie, Landnahme und Vermessung im kolonialzeitlichen Mexiko (Neuspanien)"; ${ }^{11}$ otro proyecto fue acerca de los cartógrafos que acompañaron al emperador Maximiliano a México y que permanecieron en el país como ingenieros civiles; resultado de esas investigaciones fue el libro Kaiser Maximilians kartographen in Mexiko (Cartógrafos del emperador Maximiliano en México). ${ }^{12}$

A raíz de su jubilación de la Universidad de Bayreuth en 2000 presentó una exposición en la Biblioteca de la Facultad de Economía de la Universidad Nacional Autónoma de México: Vestigios de las haciendas henequeneras de Yucatán. Documentos fotográficos de 1977 a 2004, y en 2006 otra titulada Agrimensura y cartografía en México 1720-1920 en el vestíbulo del Instituto de Geografía de la mencionada Universidad. Héctor Mendoza Vargas escribió acerca de esta última: "En los marcos de cada mapa quedaron atrapados no sólo las técnicas, escalas y datos del interés de Nickel, sino también los vestigios del cambio geográfico, las territorialidades de vastas extensiones y las resistencias sociales que aún esperan las preguntas desde las nuevas perspectivas que ofrecen la Geografía histórica y la historia de la cartografía de México" ${ }^{13}$

Los últimos años de su vida los dedicó a realizar libros electrónicos sobre las investigaciones y fotografías que realizó en México

\footnotetext{
${ }^{10}$ Publicado en Historia y Grafía, núm. 15, 2000, pp. 241-268.

${ }^{11}$ Herbert J. Nickel, "Kartographie, Landnahme und Vermessung im kolonialzeitlichen Mexiko (Neuspanien)", Ibero-amerikanisches Archiv, Neue Folge, vol. 22, núm. 3/4, 1996, pp. 421-488.

${ }^{12}$ Publicada en Frankfurt am Main, Vervuert Verlag, 2003.

${ }^{13}$ Héctor Mendoza Vargas, "Agrimensura y cartografía en México 1720-1920", Boletín, Instituto de Investigaciones Geográficas, núm. 61, 2006, p. 150 (148150).
} 
y sobre México; por ejemplo: Relikte der Henequén-Haciendas auf Yucatán aus dem 19. und 20. (Reliquias de las haciendas henequeneras en Yucatán de los siglos XIX y xx); Hacienda San José Ozumba (Puebla): die "figuritas" am alten Kornspeicher (Hacienda San José de Ozumba, Puebla: las "figuritas" de la vieja troje); Österreichische Kartographen in Mexiko, (1864-1925) (Cartógrafos austríacos en México, 1864-1925); Agrimensura y cartografía en México 1720-1920; Vestigios de las haciendas henequeras de Yucatán: documentos fotográficos para la arqueología industrial motores y cuartos de máquina; y Arbeitsverhältnisse in Henequén-haciendas aufYucatán (Condiciones de trabajo en las haciendas henequeneras de Yucatán). ${ }^{14}$

Con estas últimas investigaciones Nickel logró rescatar, por medio de la fotografía, la maquinaria utilizada en el proceso de desfibración del henequén; pudo documentar el fin de un monocultivo agroindustrial en México, sus instalaciones y los trabajadores de ese proceso.

Las investigaciones de Nickel confirmaron, al igual que las de Jan Bazant y Ursula Ewald, que la hacienda era una institución capitalista, pero también que ésta no sólo era de carácter económico sino también social, que sus propietarios establecían una relación que iba más allá de lo económico, que la deuda de

${ }^{14}$ Jahrhundert [recurso electrónico]. Freiburg i. Br., (Alemania). Arnold-Bergstraesser Institut, 2008, un disco óptico láser (Bilddokumente zur Industriearchäologie Yucatáns; 1); [Freiburg, Br.] [Arnold-Bergstraesser-Inst.], 2008, un disco óptico láser (Bilddokumente zu Haciendas im zentralmexikanischen Hochland, 1); Freiburg, Br., Arnold-Bergstraesser-Inst., 2009, un disco óptico láser; Texto y fotografía Herbert J. Nickel, México, D. F., El Colegio de México/ Universidad Nacional Autónoma de México-Centro de Investigaciones Interdisciplinarias en Ciencias y Humanidades/Consejo Nacional para la Cultura y las Artes/Consejo Nacional de Ciencia y Tecnología, 2010, un disco óptico láser; Alemania, México, Arnold Bergstraesser Institut/Universidad Nacional Autónoma de México-Centro de Investigaciones Interdiciplinarias en Ciencias y Humanidades/El Colegio de México, 2011, un disco óptico láser; Bayreuth, ABI-Verlages, 2015, un disco óptico láser (Bilddokumente zur industriearchäologie Yucatans; 7). 
los trabajadores puede estudiarse como una prestación más otorgada por el propietario para garantizar la mano de obra, y que eso puede explicar por qué los trabajadores de algunas haciendas, principalmente en la zona central del país, prefirieron permanecer en las fincas antes que formar parte de las filas de la Revolución. Pero, además, Nickel también demostró cómo los trabajadores de las haciendas recurrieron a los tribunales para defenderse de los malos tratos que les imponían sus dueños, o sus representantes, es decir, los administradores.

Los estudios de Nickel contribuyeron de manera significativa a la historia de la hacienda en México. Sus investigaciones proporcionaron nuevos enfoques e interpretaciones; su interés por romper con mitos de la historia oficial sobre esta unidad social y económica también contribuyó a conocer con mayor profundidad la historia agraria de nuestro país. Poco antes de fallecer, le fue otorgado el Reconocimiento al Investigador Extranjero del XVIII Premio Citibanamex 雼anasio G. Saravia de Historia Regional Mexicana 2018-2019. 